Compte rendu de Emmanuelle Avril, Pauline Schnapper (eds.), Le Royaume-Uni au XXI siècle : mutations d'un modèle

\title{
Baudry Rocquin
}

\section{OpenEdition}

\section{Journals}

Édition électronique

URL : http://journals.openedition.org/rfcb/877

DOI : $10.4000 / \mathrm{rfcb} .877$

ISSN : 2429-4373

Éditeur

CRECIB - Centre de recherche et d'études en civilisation britannique

Référence électronique

Baudry Rocquin, " Compte rendu de Emmanuelle Avril, Pauline Schnapper (eds.), Le Royaume-Uni au XXI siècle : mutations d'un modèle », Revue Française de Civilisation Britannique [En ligne], XXI-1 | 2016, mis en ligne le 20 juillet 2016, consulté le 01 mai 2019. URL : http://journals.openedition.org/rfcb/877

Ce document a été généré automatiquement le 1 mai 2019.

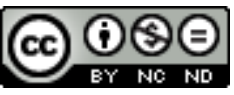

Revue française de civilisation britannique est mis à disposition selon les termes de la licence Creative Commons Attribution - Pas d'Utilisation Commerciale - Pas de Modification 4.0 International. 


\title{
Compte rendu de Emmanuelle Avril, Pauline Schnapper (eds.), Le Royaume-Uni au XXI siècle : mutations d'un modèle
}

\author{
Baudry Rocquin
}

\section{RÉFÉRENCE}

Emmanuelle AVRIL, Pauline SCHNAPPER (eds.), Le Royaume-Uni au XXI siècle : mutations d'un modèle, Paris : Editions Ophrys, 2014, 374 p., ISBN : 978-2-7080-1378-0, 25 euros.

1 Le livre coordonné par Emmanuelle Avril et Pauline Schnapper est une contribution importante à la science politique, la sociologie et l'histoire récente du "modèle» britannique au XXI siècle.

2 Peut-on encore parler aujourd'hui d'un modèle britannique ? Par modèle on entend un exemple qu'on admire, qu'on recopie et qui s'exporte. C'était le cas à partir du XVIII et surtout du XIX ${ }^{\mathrm{e}}$ siècle où le Royaume-Uni était perçu comme un modèle de démocratie parlementaire, de développement industriel et de puissance mondiale à travers son Empire dont s'inspiraient de nombreux pays, alimentant ainsi le mythe d'un « exceptionnalisme britannique ».

3 L'introduction distingue trois grands traits de ce modèle: le modèle politique de Westminster, le rôle de l'Etat dans l'économie et le pluralisme et la tolérance religieuse du point de vue social. Globalement, ce qui caractérisait ce «modèle » britannique depuis 1945 a été sa souplesse et son succès qui ont permis des changements progressifs politiques tout en assurant une certaine cohésion sociale, appuyés sur sa lingua franca qui l'ont imposé dans le monde.

4 Les mutations mises en avant dans l'ouvrage sont de deux types: des transformations internationales - la mondialisation des échanges, les changements technologiques, 
l'entrée dans la Communauté européenne, la fin de la guerre froide, les attaques du 11 septembre 2001 - et des évolutions internes - les évolutions démographiques, le maintien de fortes inégalités sociales et géographiques, la crise de légitimité du système politique, l'émergence d'un gouvernement de coalition conservateur-libéral démocrate en 2010, la menace de l'indépendance de l'Ecosse, la crise financière et économique, les critiques du multiculturalisme depuis 2001.

5 Tous ces développements contribuent à remettre en question la souplesse du modèle britannique dans un monde en changement. Ce sont ces questions, et en particulier celui des mutations de l'exceptionnalisme britannique, que les auteurs de cet ouvrage soulèvent - avec beaucoup de talent - en abordant successivement les aspects politiques, économiques, sociaux et culturels du Royaume-Uni au début du XXI ${ }^{e}$ siècle.

Dans la première partie, consacrée aux questions politiques et institutionnelles, les auteurs montrent une adaptabilité des institutions qui ont toutes évolué, notamment sous l'impulsion du New Labour de Tony Blair (réforme de la chambre des Lords, « relation spéciale ») et des idéologies qui ont également changé, avec une normalisation des petits partis et un affaiblissement des élections transformées par le multipartisme. Enfin, la politique étrangère britannique a du mal à évoluer, malgré l'expérience du déclin. Un des grands atouts de cette partie est de présenter les partis politiques (Conservateurs, Travaillistes mais aussi libéraux-démocrates, SNP, Plaid Cymru, UKIP, etc.) sans partipris, avec autant de précision et de développement pour chacun, de manière à bien montrer l'émergence d'une « opposition » multipartite.

7 La deuxième partie traite des aspects économiques et sociaux et met en lumière une évolution graduelle vers une libéralisation de l'économie (des services et de la finance, principalement) et de la société (droits des femmes, des homosexuels mais aussi évolution du financement des universités ou du NHS), reflétant une tradition de libéralisme social proprement britannique. Ce phénomène s'accompagne paradoxalement de la persistance des inégalités économiques, géographiques et sociales, de la polarisation traditionnelle entre nord et sud, entre haves et have nots. Cette partie est particulièrement habile à mettre en évidence les complexes réformes sociales-libérales depuis le New Labour dans la société, que ce soit dans l'enseignement, la santé, la justice ou l'économie, et qui jouent de l'identité britannique au $\mathrm{XXI}^{\mathrm{e}}$ siècle. Ces domaines sont-ils indépendants des changements politiques et mondiaux?

8 La troisième partie, qui couvre les aspects culturels, traduit la même ambivalence, entre l'émergence d'un modèle d'intégration, notamment des minorités ethniques et religieuses, et la persistance de clivages socio-culturels qui dans certains cas semblent de plus en plus marqués. On note là encore que l'identité britannique, au travers du sport comme le rugby ou les Jo de 2012 ou le rôle des médias papier en déclin, évolue vers plus de périphéries. On l'observe également dans les loisirs ou le mainstreaming du cinéma britannique.

9 Cet ouvrage est donc un parfait manuel de science politique, sociologie ou histoire contemporaine du Royaume-Uni, qui satisfera tout autant les chercheurs en civilisation britannique que les étudiants en abordant de manière précise et exhaustive de nombreux domaines de la culture et de l'histoire récente du Royaume-Uni. La lecture est d'autant plus stimulante que les auteurs ont réussi le pari de réunir plus d'une dizaine de contributeurs spécialistes dans leur domaine (dont Jean-Claude Sergeant, à qui le livre est dédié) en un peu plus de 300 pages avec chiffres et faits à la clé, tout en assurant une homogénéité des sections surprenante. Il semble que le livre ait été écrit d'une seule 
main. Enfin, et ce n'est pas négligeable pour un ouvrage de civilisation britannique, il contient très peu de calques et propose très souvent une traduction des concepts importants en français tout en se référant aux termes originaux en langue anglaise entre parenthèse dans le texte. Cela en fait un ouvrage tout à fait homogène et agréable à lire. Chaque chapitre dispose ainsi d'une bibliographie à jour et concise dans chacune des sections. Ce choix permettra autant aux non-spécialistes qu'aux étudiants de parfaire leurs connaissances auprès de textes plus spécialisés sans pour autant nécessiter de l'érudition. Les chercheurs quant à eux trouveront leur bonheur dans les mises à jour des questions économiques, politiques et sociales depuis 2010 et des problématiques sur l'identité britannique au XXI siècle abordée à travers de nombreuses perspectives - du sport à l'économie en passant par la «relation spéciale ». Les derniers ajouts théoriques sont présents, notamment sur les questions internationales et sociales (le «Great British Class Survey » de Savage et Devine de 2013, par exemple).

11 Pour terminer, il faut souligner la qualité du cahier couleur en fin d'ouvrage et la présence d'un index - certes un peu court par rapport au nombre de concepts évoqués dans le corps du texte - qui parachèvent cette œuvre qui fera date dans les questions de modèles nationaux et d'identité britannique. On attend d'ores et déjà avec impatience la seconde édition qui, après les élections du 7 mai 2015 et ce qui suivra, permettront de préciser encore celles-ci.

\section{AUTEURS}

\section{BAUDRY ROCQUIN}

Université de Bordeaux, Centre Emile Durkheim (UMR 5116) 